\section{Fatores associados à ocorrência de anemia em crianças menores de seis anos: uma revisão sistemática dos estudos populacionais}

\section{Associated factors of anemia in children under six years old: a systematic review of the population studies}

Luciana Pedrosa Leal 1

Mônica Maria Osório 2

\author{
${ }^{1}$ Departamento de Enfermagem. Centro de Ciências da Saúde. \\ Universidade Federal de Pernambuco. Av. Prof. Moraes Rego, \\ 1235. Cidade Universitária. Recife, PE, Brasil. CEP: 50.670-901. \\ E-mail: lucianapleal@hotmail.com \\ 2 Departamento de Nutrição. Centro de Ciências da Saúde. \\ Universidade Federal de Pernambuco. Recife, PE, Brasil.
}

\begin{abstract}
Objectives: to review the articles published between 1997 and 2010 with representative population samples for children under six years, estimating prevalence of anemia and/or iron deficiency and their associated factors by laboratorial analyses of hemoglobin or ferritin.

Methods: articles were systematically reviewed using the following keywords searched on the PubMed, SciELO and LILACS databases: anemia, iron-deficiency; anemia, iron-deficiency/epidemiology; anemia, iron-deficiency/etiology; child, preschool e risk factors.

Results: factors which presented statistical association to anemia/iron-deficiency included: children's age, maternal scholar, familiar income, diarrhea, geographic area, household wealth index, birth weight, height/age indicator, weight/height indicator, ethnics, sex, iron density and calories from cow milk. Children's age was a common factor in the international and national studies. Maternal scholar and socioeconomics and environment factors showed association with anemia in the development regions studies. Overweight and passive smoking appear as slow investigated variables.

Conclusions: study analyzes shows the multiplicity of anemia/iron-deficiency associated factors at different contexts.
\end{abstract}

Key words Anemia, Child, Risk factors, Iron deficiency

\section{Resumo}

Objetivos: revisar os artigos publicados de 1997 a 2010, com amostras populacionais representativas para crianças menores de seis anos, que estimaram a prevalência da anemia elou deficiência de ferro e seus fatores associados, por meio da análise laboratorial de hemoglobina ou ferritina.

Métodos: os artigos foram revisados sistematicamente, utilizando as palavras-chaves: anemia, irondeficiency; anemia, iron-deficiency/epidemiology; anemia, iron-deficiency/etiology; child, preschool e risk factors nas bases de dados PubMed, SciELO e LILACS.

Resultados: os fatores mais citados foram: idade da criança, escolaridade materna, renda familiar, diarréia, área geográfica, índice de riqueza do domicílio, peso ao nascer, indicador altura/idade, indicador peso/altura, etnia, sexo, densidade de ferro e calorias provenientes do leite de vaca. A idade da criança foi comum nos estudos nacionais e internacionais. A escolaridade materna e fatores socioeconômicos e ambientais mostraram associação com a anemia nos estudos realizados nas regiões em desenvolvimento. O sobrepeso e a exposição passiva ao fumo surgem como variáveis pouco investigadas.

Conclusões: a análise demonstra a multiplicidade de fatores associados à anemia/deficiência de ferro existentes em diferentes contextos.

Palavras-chave Anemia, Criança, Fatores de risco, Deficiência de ferro 


\section{Introdução}

A anemia e a deficiência de ferro se constituem atualmente em um desafio à saúde pública, uma vez que essas carências são as desordens nutricionais mais comuns do mundo e diversos fatores (biológicos, socioeconômicos, ambientais, de saúde, nutrição, etc), isolados ou em interação, podem contribuir para o seu aparecimento. 1,2 No caso do Brasil, a análise da transição nutricional demonstra que a prevalência de anemia continua elevada em contraste com o declínio da desnutrição nas crianças, tornando-se, sem dúvida, o principal problema carencial do país. ${ }^{3}$

As complicações advindas da exposição à anemia, que tem as crianças menores de seis anos como um de seus grupos mais afetados, trazem conseqüências severas para a saúde infantil, como o déficit no desenvolvimento cognitivo e motor, maior susceptibilidade às infecções, entre outras.4,5

A complexidade da anemia e a dificuldade de seu controle no mundo fazem com que vários estudos busquem identificar as razões para as altas prevalências e seus potenciais fatores de risco. Por outro lado, os programas de intervenção criados com base nestas prevalências, voltados à prevenção e tratamento por meio da suplementação medicamentosa e fortificação de alimentos, parecem não ser efetivos, tendo em vista não considerar a contribuição de outros fatores externos que possam atuar sobre o problema.6-9 Assim, uma melhor identificação da anemia e dos seus fatores determinantes contribui para determinar meios efetivos para a sua solução, ocasionando a redução desta carência e de seus fatores ou interrompendo a sua associação com os efeitos adversos para a saúde.2,10

Portanto, torna-se relevante conhecer a etiologia do problema no intuito de reestruturar ações de prevenção e combate à anemia, visando proporcionar uma melhoria da saúde e nutrição da população. Neste contexto, o propósito desse artigo é realizar uma revisão de publicações no período de 1997 a 2010, que avaliem os fatores associados à anemia e/ou deficiência de ferro em crianças menores de seis anos de idade.

\section{Métodos}

O presente estudo é uma revisão sistemática visando avaliar estudos epidemiológicos de corte transversal segundo as diretrizes do Manual Cochrane dos revisores adotados pela Universidade Federal de São Paulo, 11 e Preferred Reporting Items for Systematic reviews and Meta-Analyses (PRISMA). 12
A pesquisa foi realizada nas bases de dados da U.S. National Library of Medicine and the National Institutes Health (PubMed) e da Biblioteca Virtual em Saúde (BVS), utilizando as referências da Scientific Eletronic Library Online (SciELO) e da Literatura Latino-Americana e do Caribe em Ciências da Saúde (LILACS), usando os seguintes termos (DeCS/MeSH): 13 "anemia, iron-deficiency"; "anemia, iron-deficiency/epidemiology"; "anemia, iron-deficiency/etiology"; "child, preschool" e "risk factors" na base de dados PubMed; e "anemia, child e risk factors" na BVS.

A identificação e seleção dos estudos foram realizadas por dois pesquisadores, de forma independente, considerando como critérios de inclusão, artigos publicados entre 1997 e 2010, oriundos de estudos transversais com amostras populacionais para crianças menores de seis anos, que investigaram os fatores associados e/ou determinantes da anemia e/ou deficiência de ferro, por meio de análise laboratorial da hemoglobina ou da ferritina, ${ }_{1}$ com análise de regressão múltipla, em idioma inglês ou português. Estudos pontuais específicos de instituições como creches, escolas e unidades de saúde foram excluídos. Artigos aparecendo em mais de uma base de dados, foram computados em apenas uma base, considerando a PubMed e SciELO, nesta ordem, como prioridades.

A qualidade metodológica dos estudos foi avaliada pelos dois pesquisadores considerando os seguintes itens: clareza e adequação na descrição do processo de amostragem; randomização da amostra; especificação dos critérios de inclusão e exclusão; descrição das perdas e exclusões e apropriada apresentação dos resultados. No caso de discordância entre os pesquisadores a permanência de um artigo neste estudo foi decidida por consenso após revisão dos critérios de inclusão, e reanálise do texto.

Publicações de dados de uma mesma pesquisa foram identificadas em dois momentos durante a seleção dos estudos. Nestes casos, 14-17,22,24,28 os estudos foram incluídos na revisão por se entender que as análises possuíam objetivos, amostras e métodos de análise estatística diferentes. Contudo, os fatores associados e/ou determinantes da anemia e/ou deficiência de ferro, constantes em diferentes estudos resultantes de um mesmo banco de dados foram computados na análise uma única vez.

\section{Resultados}

$\mathrm{Na}$ base PubMed foram identificadas inicialmente 144 artigos e conforme os títulos que citavam os descritores definidos, foram selecionadas 58. Após a 
leitura dos artigos correspondentes a estas 58 referências foi realizada uma segunda seleção, de acordo com os critérios de inclusão, restando 9 artigos.

Na base de dados da Biblioteca Virtual em Saúde (BVS), foram identificadas inicialmente 25 referências, sendo 14 no SciELO e 11 no LILACS. A primeira seleção pelos títulos, resultou em 11 referências no SciELO e 1 no LILACS. Na segunda seleção, após a leitura dos artigos correspondentes às referências, foram incluídos 4 artigos do SciELO no presente estudo.

Além dos artigos encontrados na PubMed e na BVS, foram incluídos 4 outros citados nas referên- cias dos artigos já selecionados, e que não tinham sido encontrados nas bases de dados pesquisadas, totalizando, assim para esta revisão 17 artigos. A descrição do processo de seleção dos estudos encontra-se sintetizada na Figura 1.

Dos artigos incluídos nesta revisão, sete ${ }^{14-20}$ são estudos realizados no Brasil e dez $21-30$ em outros países. As principais características dos estudos (autores, ano de publicação e local do estudo, desenho do estudo e amostragem, amostra, diagnóstico de anemia e/ou de deficiência de ferro, variáveis analisadas e fatores associados) podem ser observadas nas Tabelas 1 e 2 .

\section{Figura 1}

Fluxograma de seleção dos artigos.

Literatura Pesquisada

Limites: - estudos transversais com amostras representativas populacionais de crianças $<6$ anos; - avaliação dos fatores determinantes da anemia elou defíciência de ferro:

- uso da hemoglobina ou ferritina para diagnóstico da anemia elou defíciência de ferro;

- período: 1997-2010;

- idioma: português ou inglês;

- análise estatística: análise múltipla.

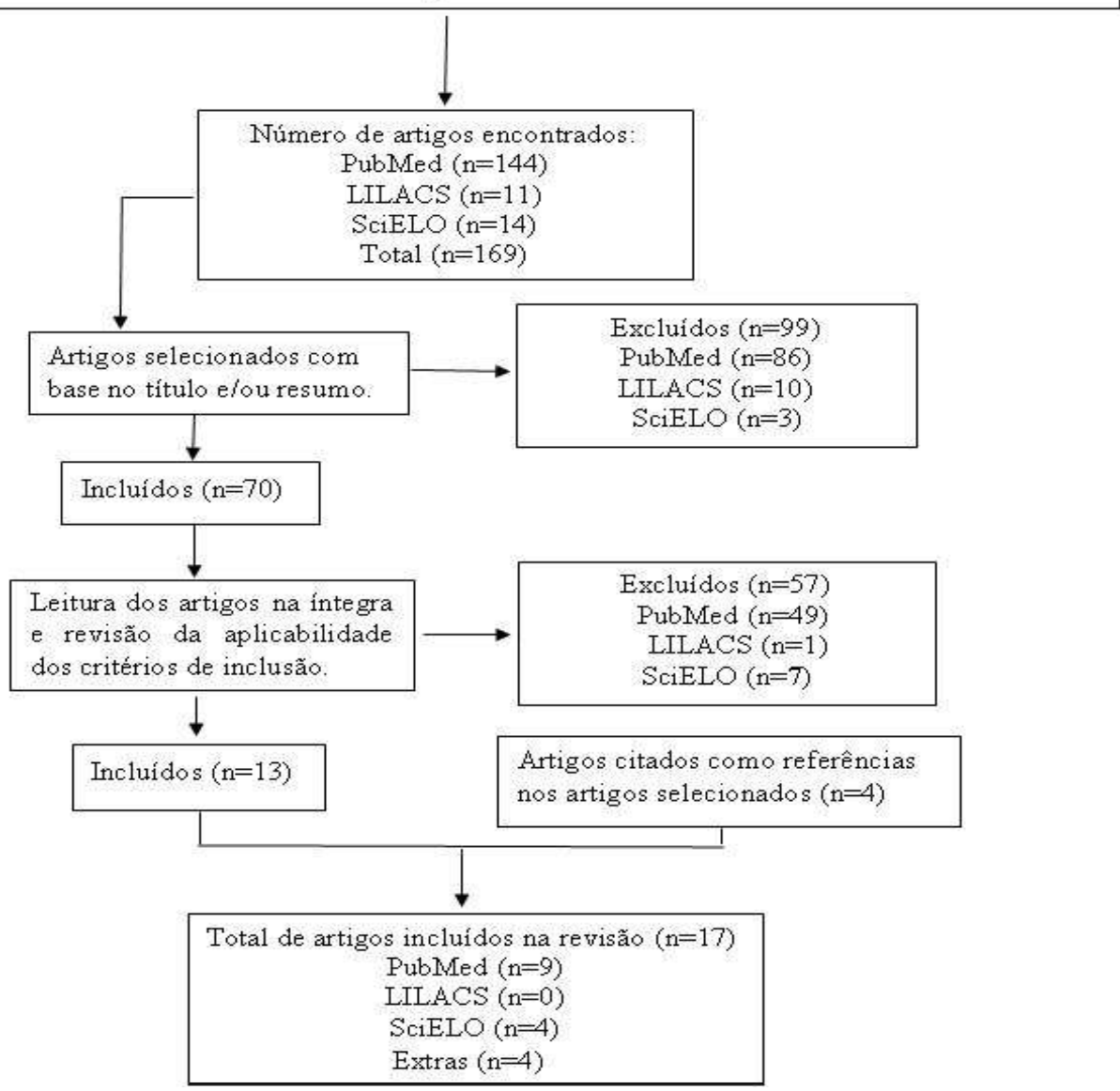




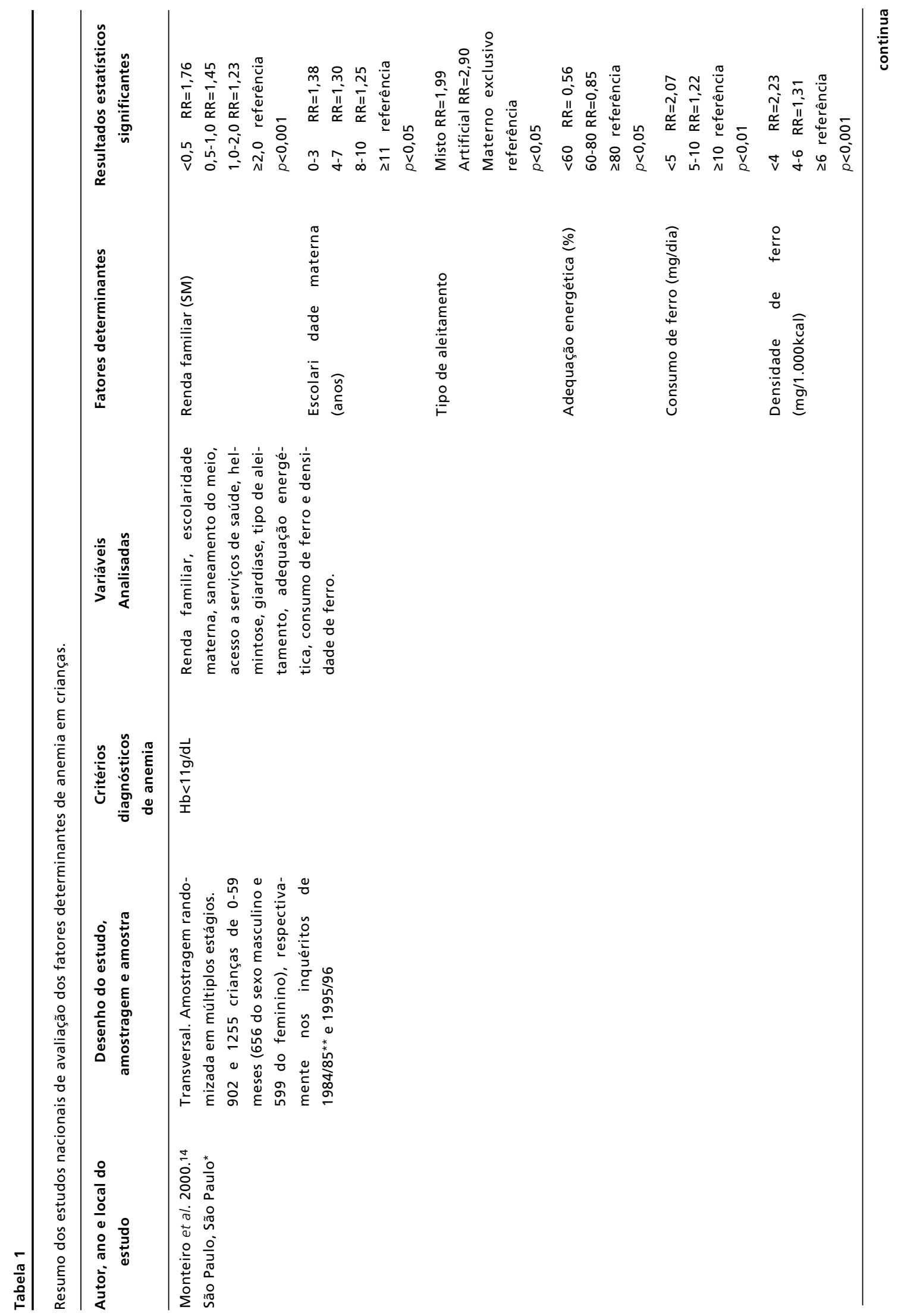




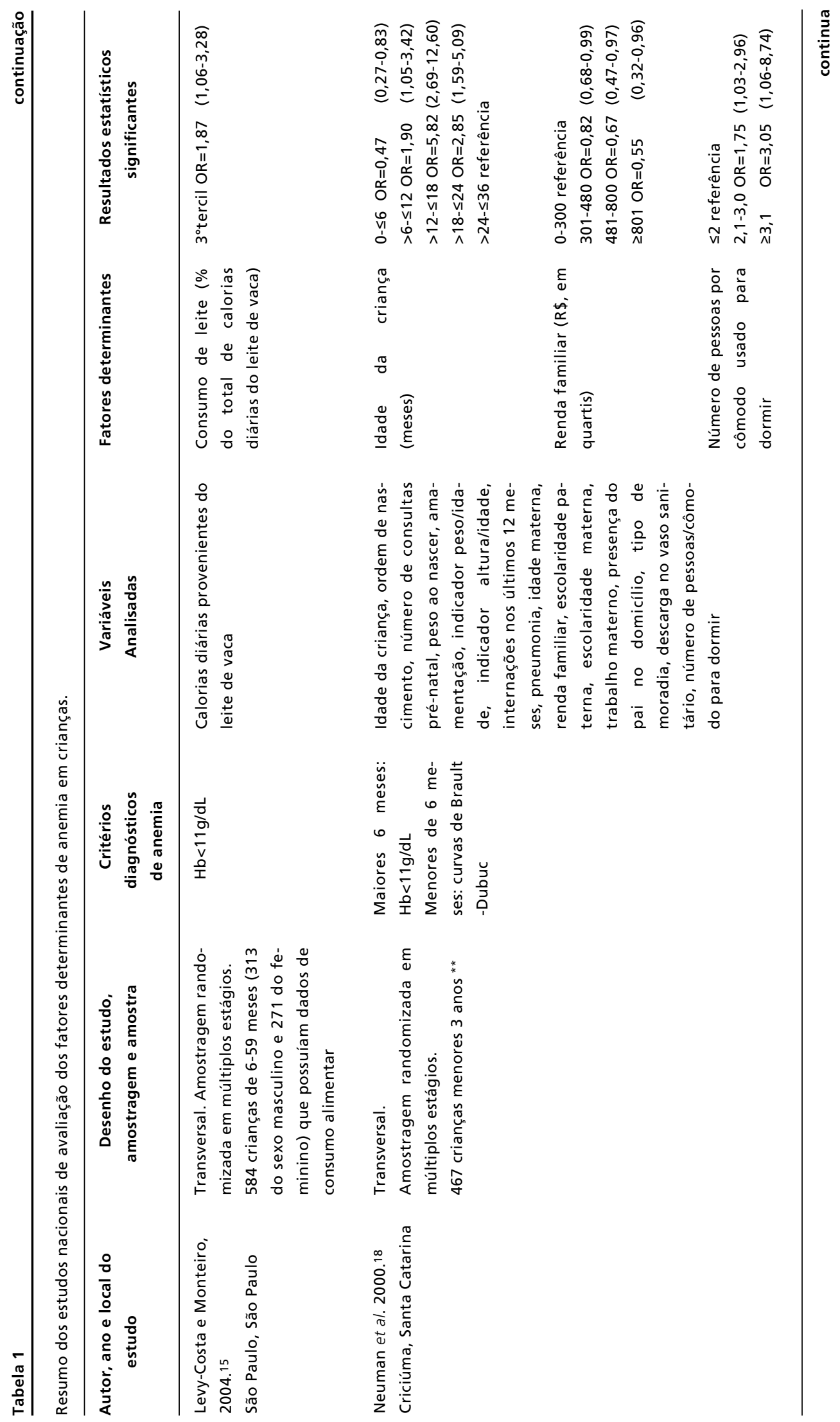




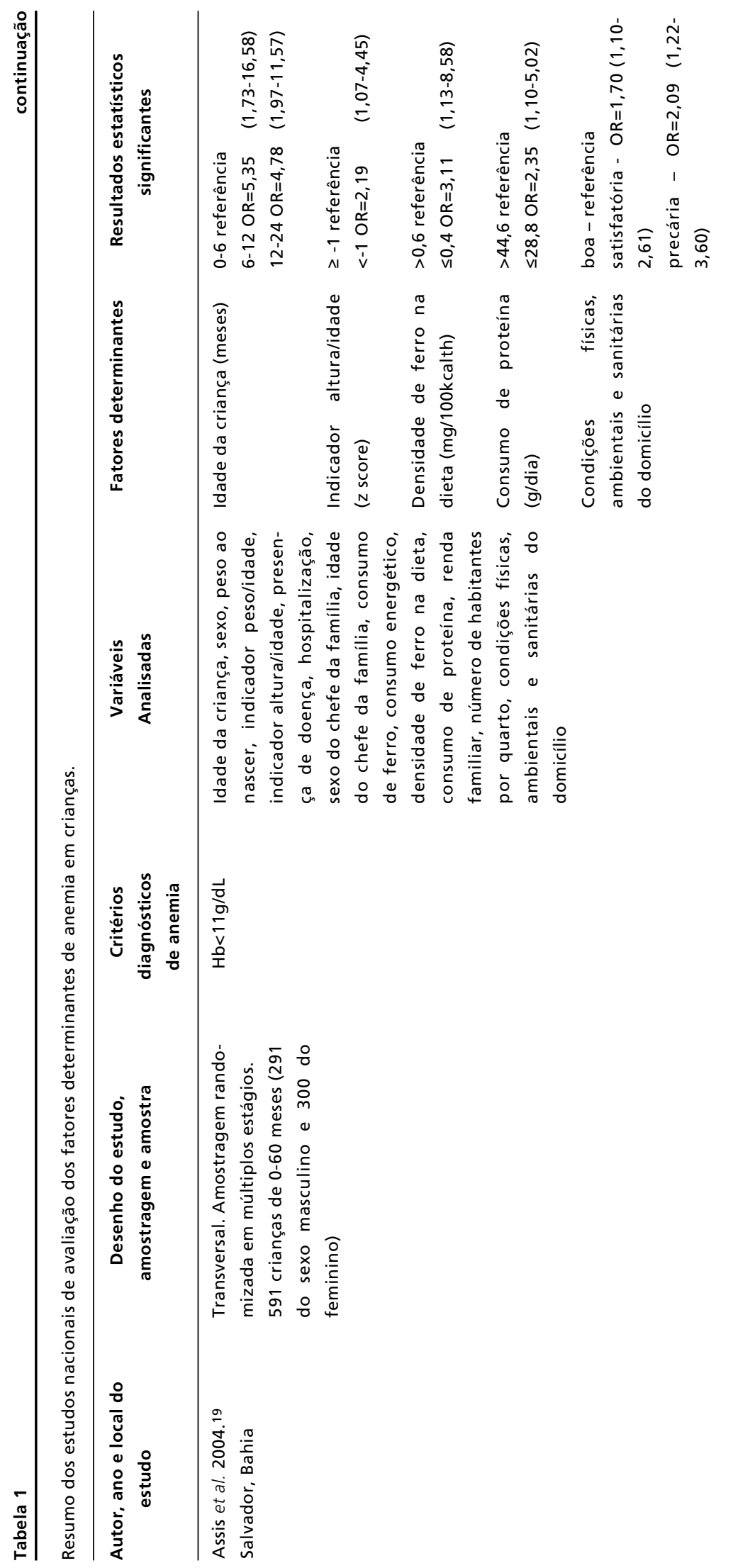




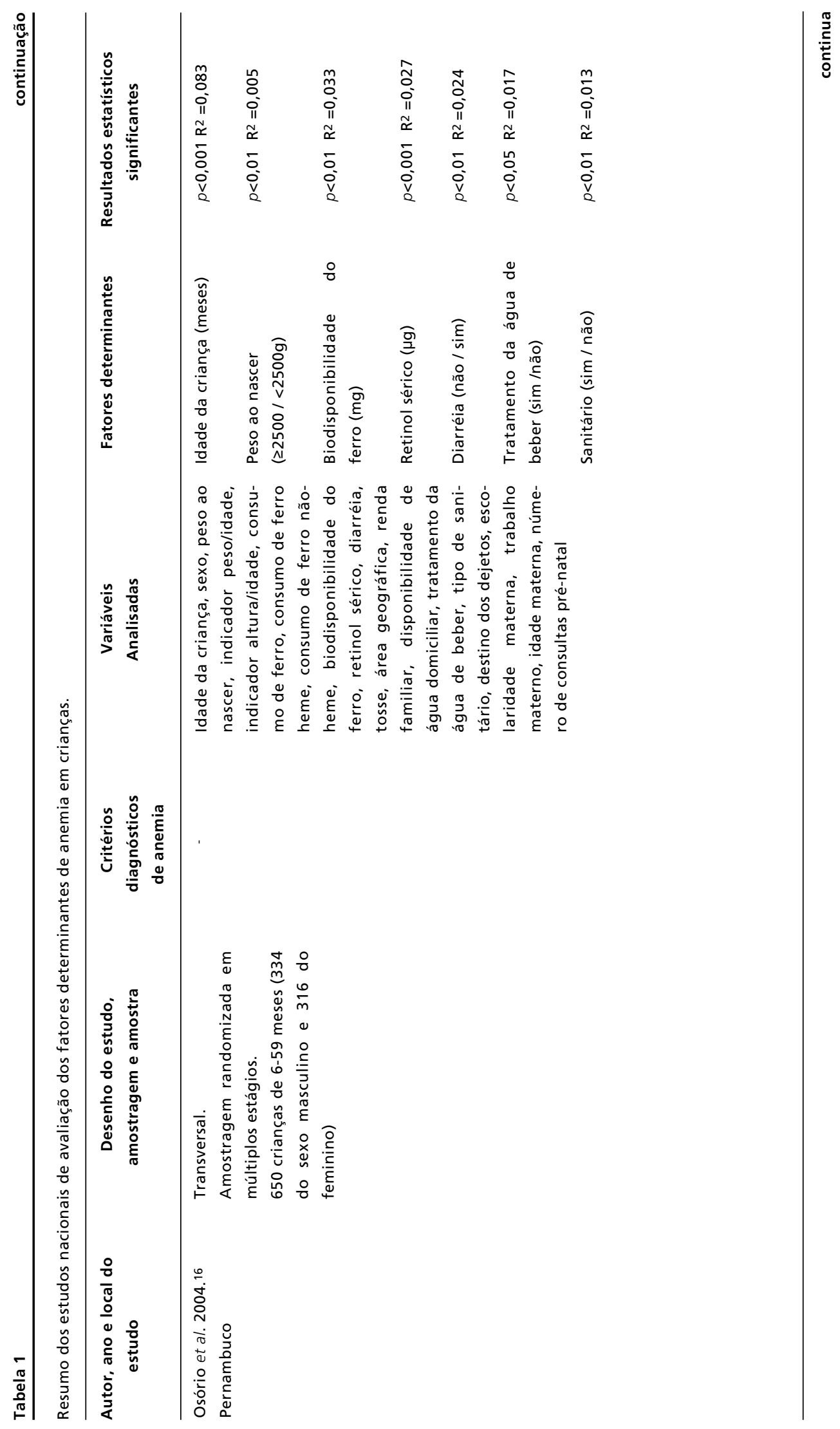




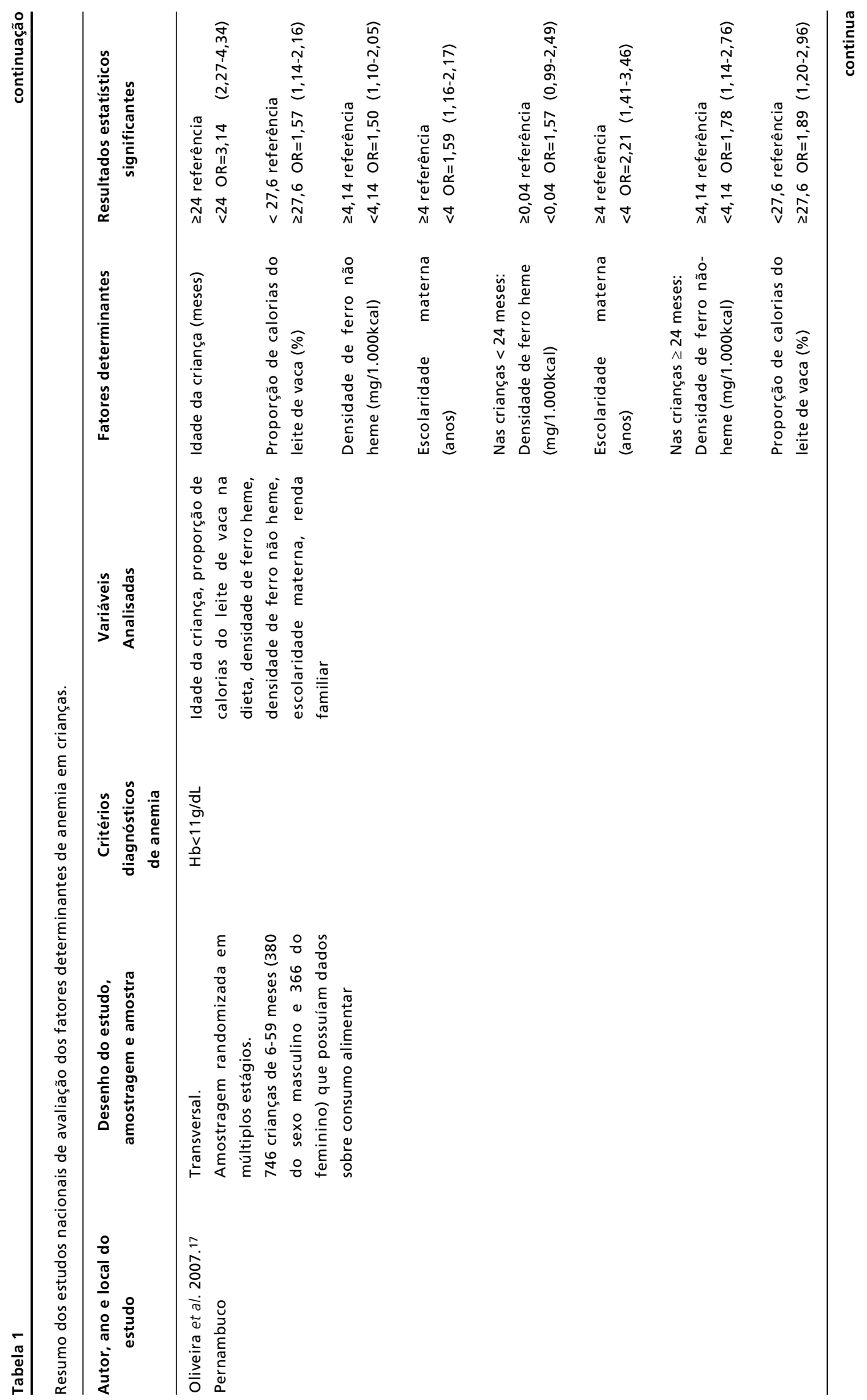




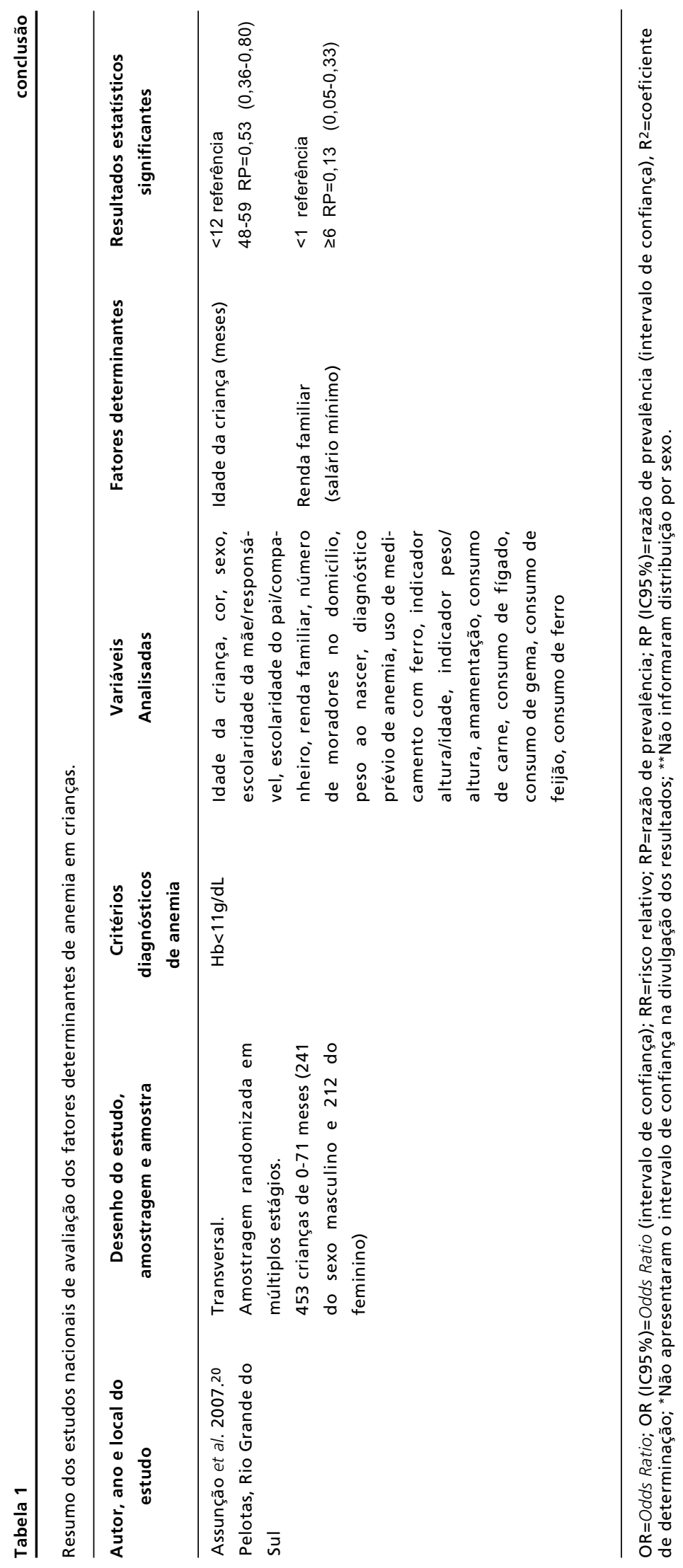




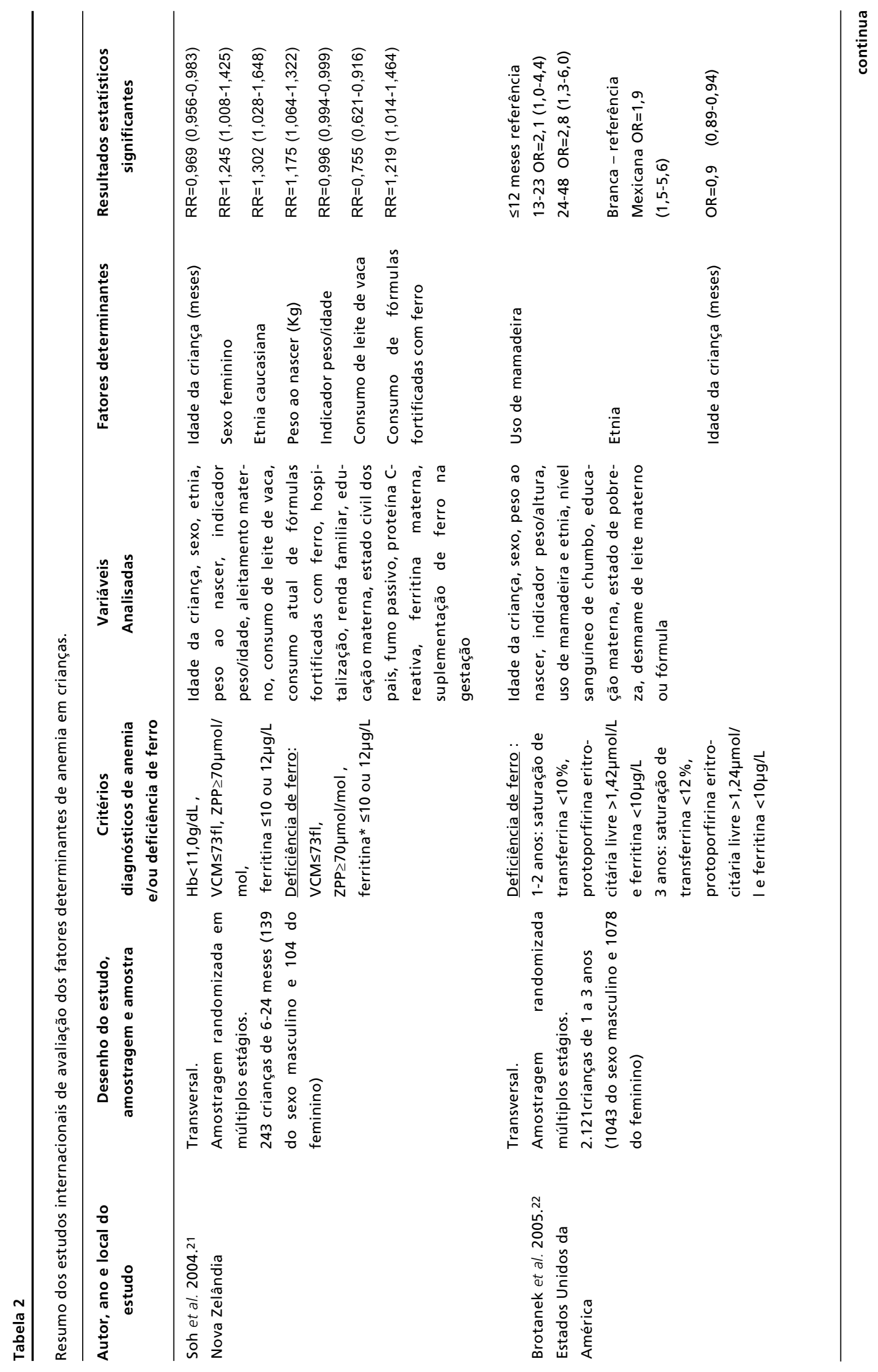




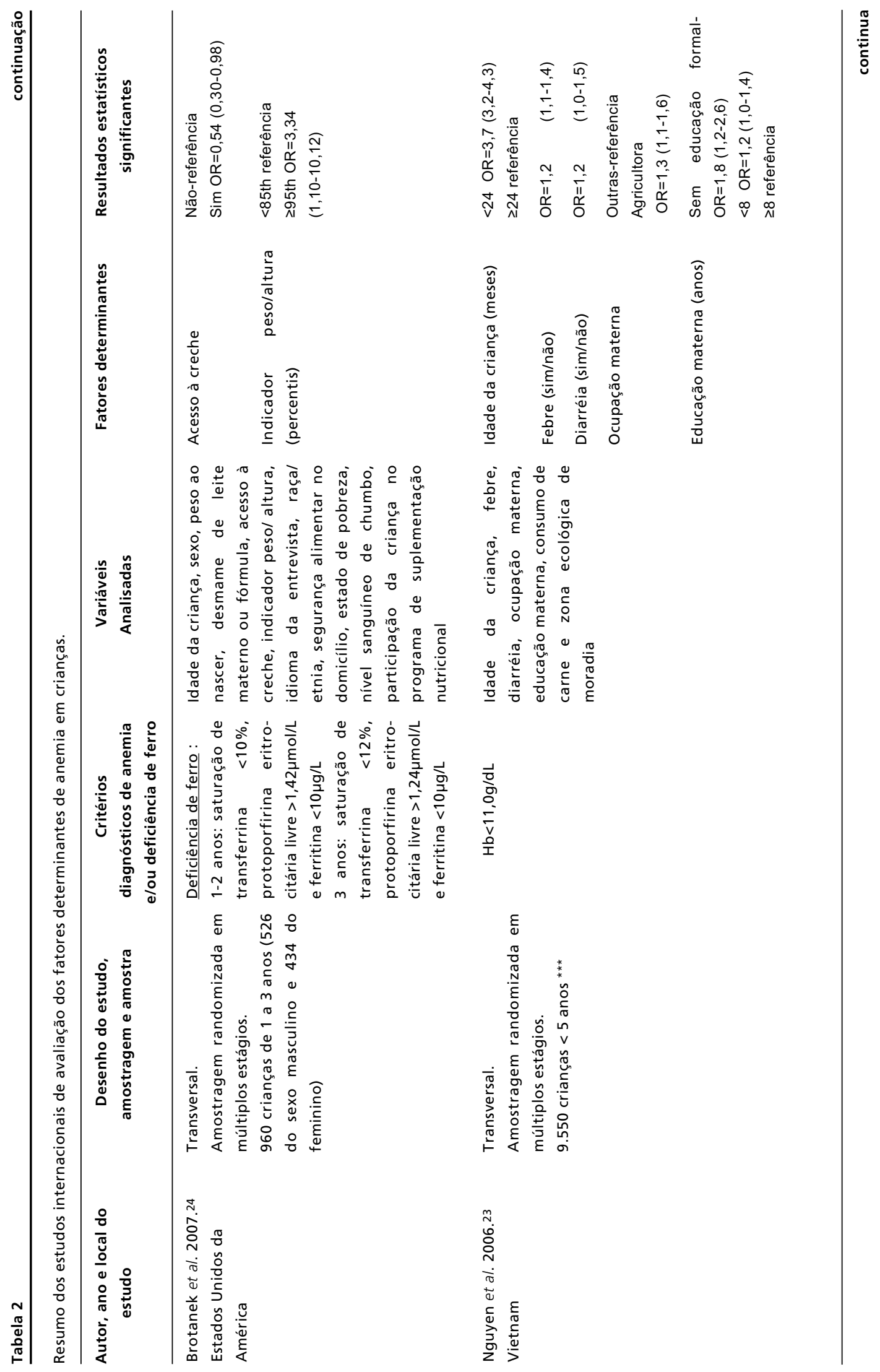




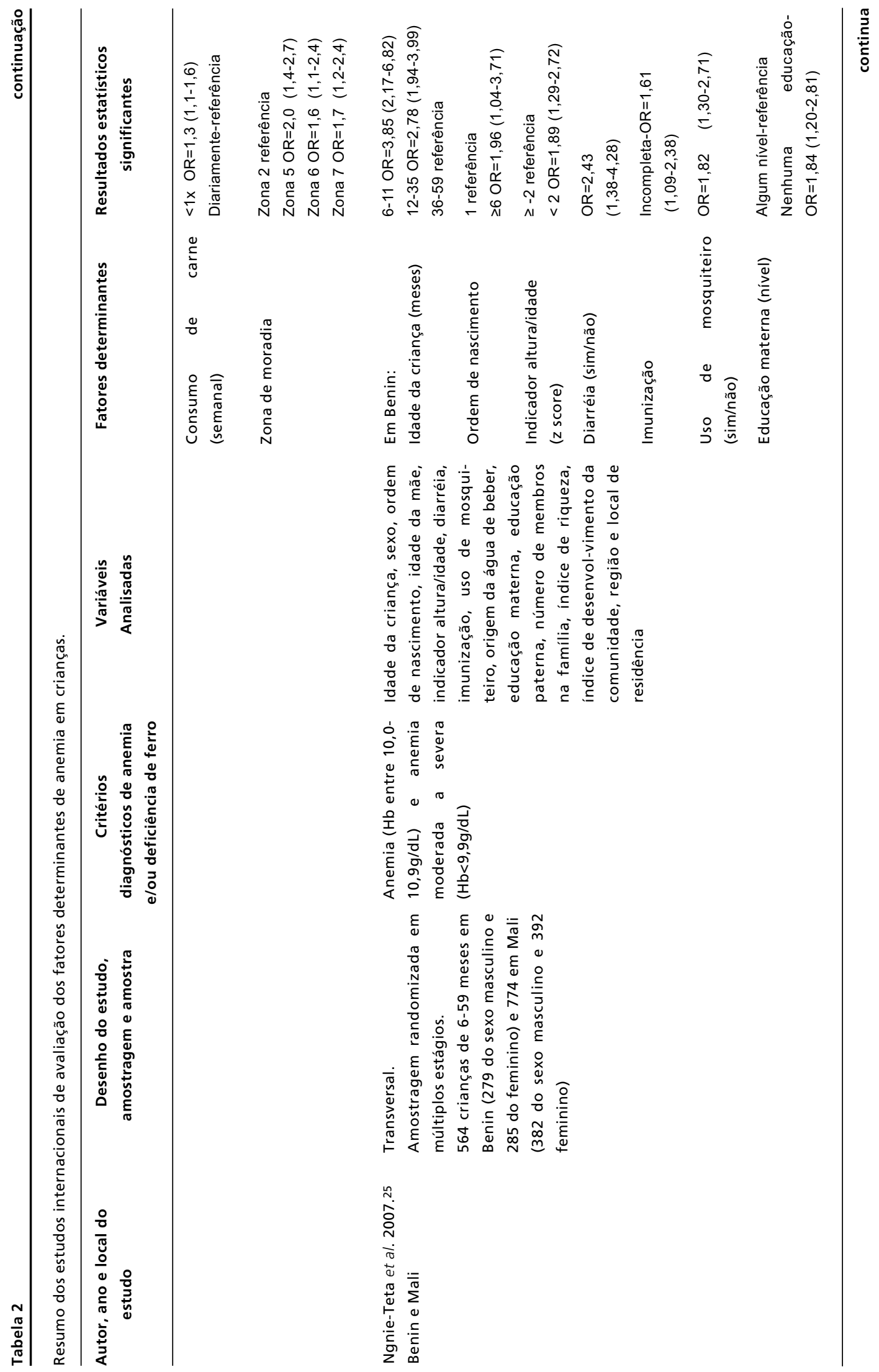




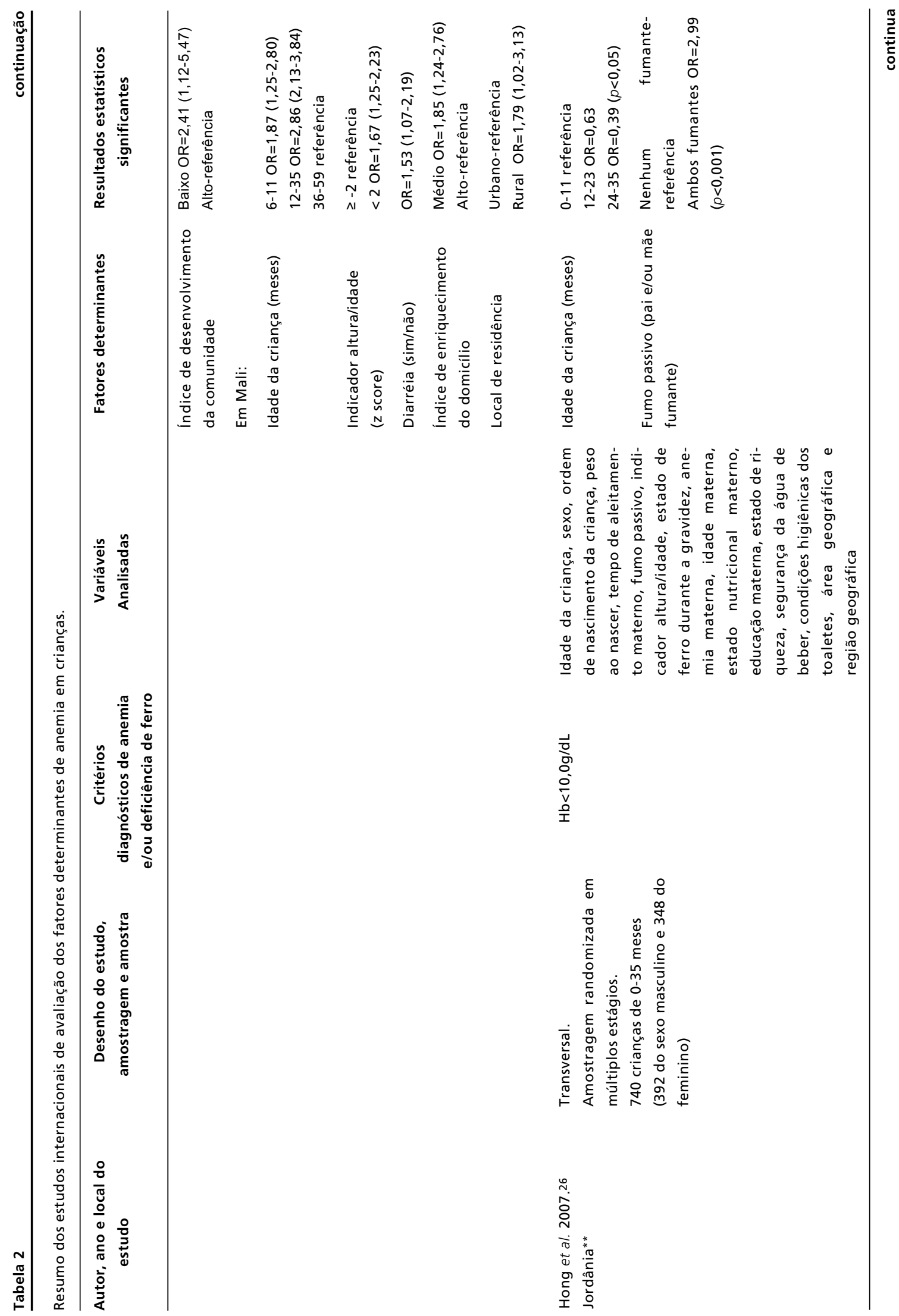




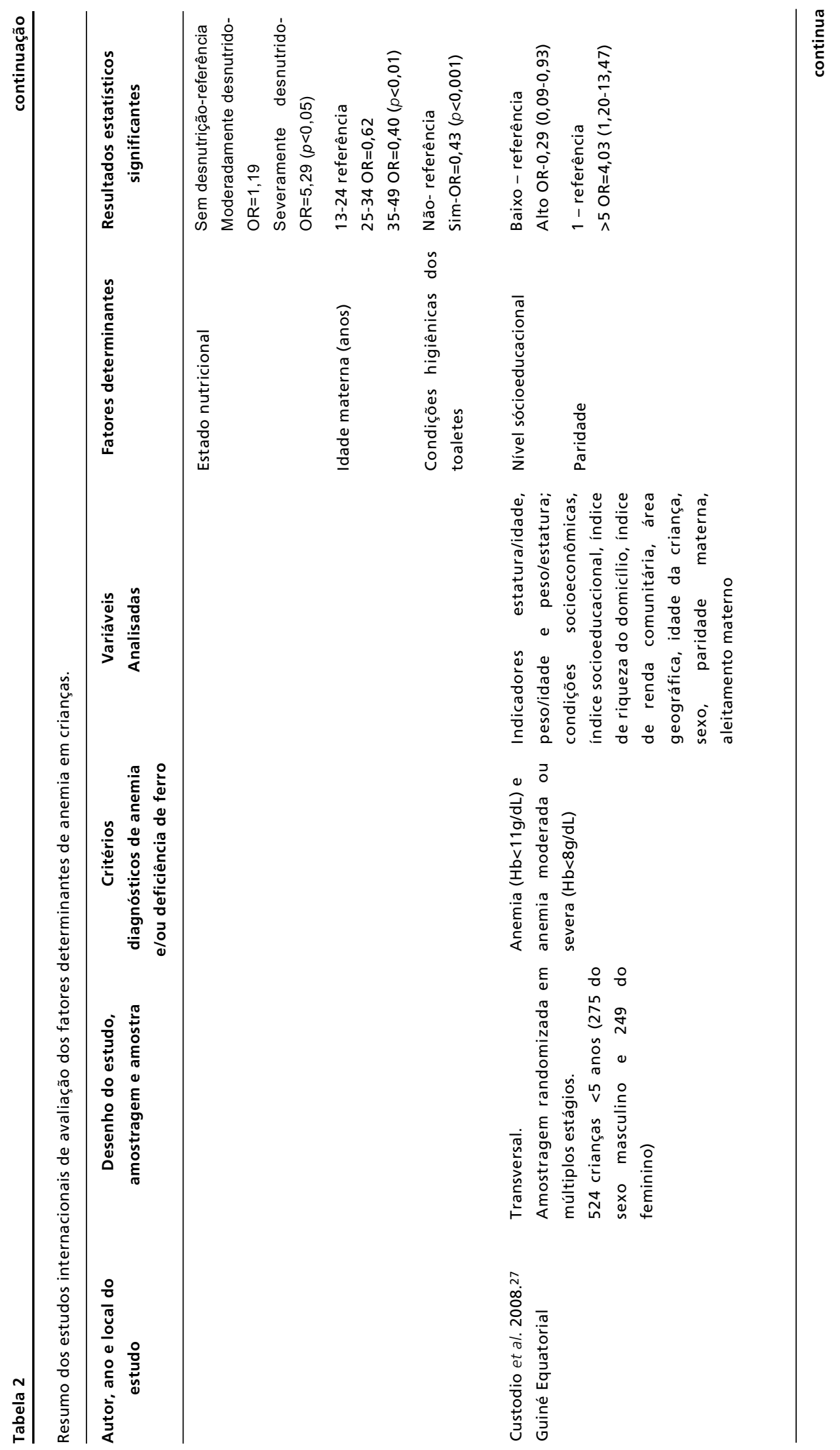




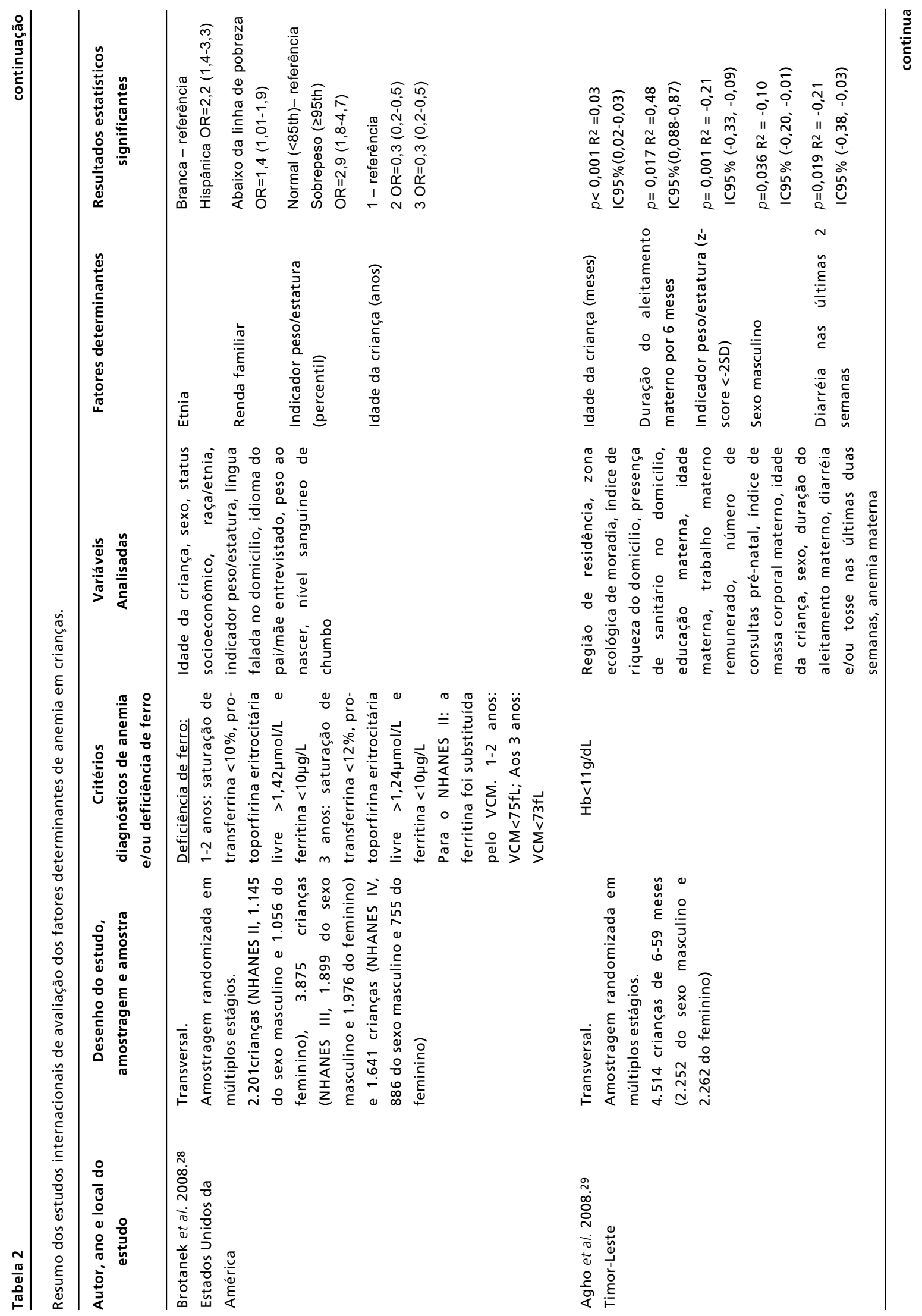




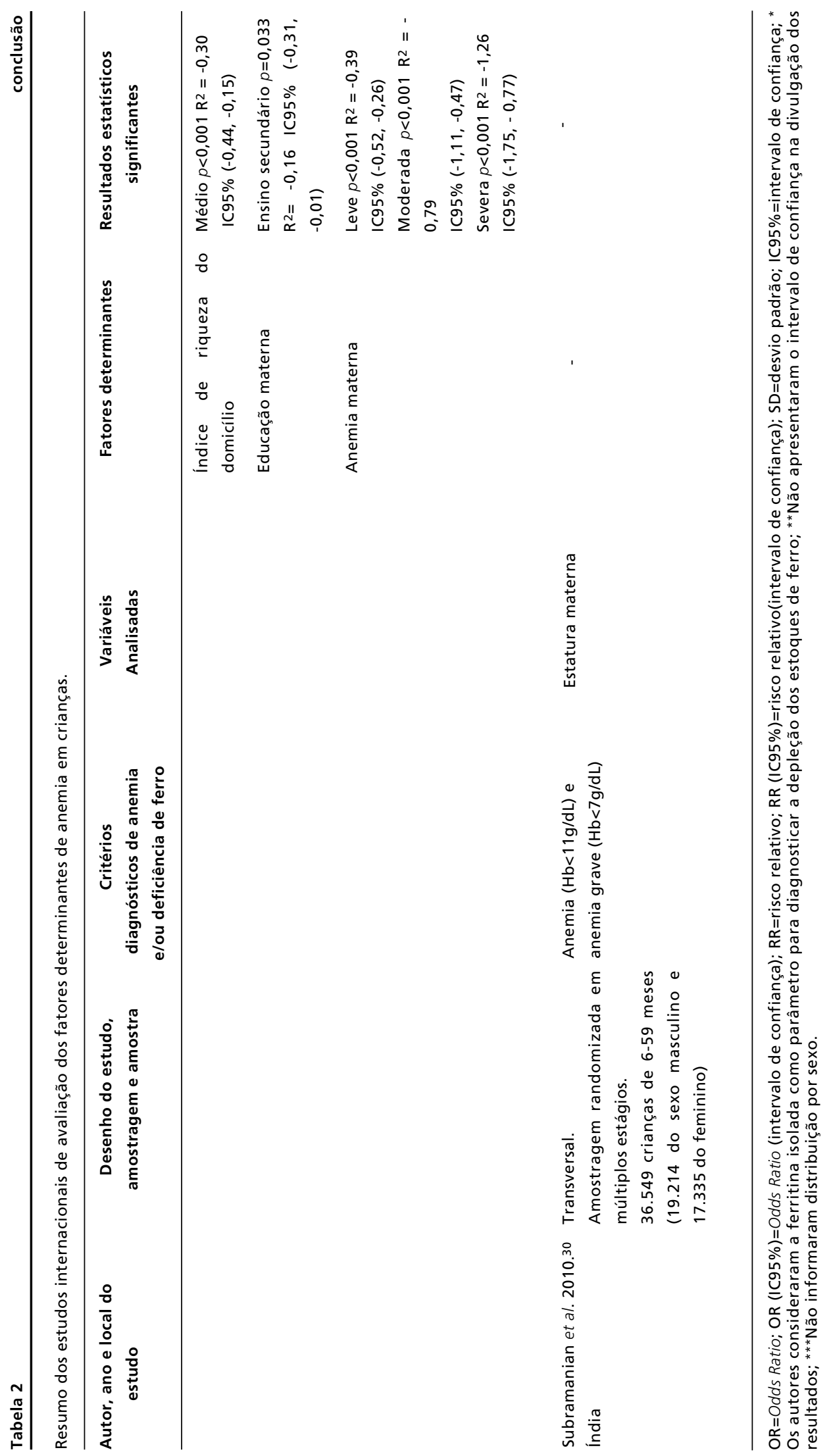




\section{Estudos realizados no Brasil}

Em um estudo sobre a tendência secular da anemia na infância na cidade de São Paulo, Monteiro et al. ${ }^{14}$ compararam dois inquéritos transversais realizados em 1984/85 e 1995/96 com amostras probabilísticas de 902 e 1256 crianças de 0-59 meses, respectivamente, selecionadas a partir de uma amostragem estratificada de múltipla etapa envolvendo o sorteio de setores censitários, conglomerados de domicílios e domicílios individuais. Os autores encontraram uma tendência crescente de prevalência de anemia em crianças de 0-59 meses variando de 35,6\% em $1984 / 85$ para $46,9 \%$ em 1995/96. Neste último inquérito a prevalência de anemia por sexo foi de $51,9 \%$ para o sexo masculino e $41,4 \%$ para o feminino. Em uma base combinada dos dados dos dois inquéritos, foram realizadas análises de regressão logística, identificando baixa renda familiar, menor escolaridade materna, aleitamento artificial, inadequação energética, menor consumo de ferro e baixa densidade de ferro, como fatores de risco de anemia. Entretanto, ao avaliarem a variação esperada na prevalência de anemia entre os inquéritos, indicando possivelmente uma melhora desta carência, isto não ocorreu.

Levy-Costa e Monteiro, 15 utilizando os dados de consumo alimentar de todas as crianças de 6-23 meses e de uma a cada três crianças de 24-59 meses do inquérito de 1995/96 do estudo acima citado,14 verificaram, em 584 crianças de 6-59 meses, a influência exercida pelo consumo de leite de vaca sobre a concentração de hemoglobina e o risco de anemia nessa população. A anemia $(\mathrm{Hb}<11 \mathrm{~g} / \mathrm{dL})$ foi diagnosticada em $45,2 \%$, sendo $49,8 \%$ no sexo masculino e $39,8 \%$ no sexo feminino. Uma regressão linear mostrou associação significante entre a concentração da hemoglobina e a percentagem do total de calorias diárias provenientes do leite de vaca, independentemente das características sóciodemográficas das crianças. No entanto, esta correlação não foi independente da densidade de ferro heme e não heme da dieta. Regressão logística evidenciou associações positivas entre a percentagem do total de calorias diárias provenientes do leite de vaca e o risco de anemia quando ajustado pelo sexo, idade e variáveis socioeconômicas e quando ajustados por estas variáveis e pela densidade de ferro na dieta.

Com o objetivo de medir a prevalência de anemia e avaliar os fatores de risco, Neuman et al. 18 estudaram uma amostra de 467 crianças menores de 3 anos na área urbana do Município de Criciúma, em Santa Catarina. A amostragem foi realizada em múltiplo estágio, incluindo divisão dos setores censitários em dois estratos: no primeiro foram visitados $54 \%$ dos domicílios de cada setor e no segundo, 155 domicílios por setor, em um total de $20 \%$ dos setores deste estrato. Foi utilizado um fator de ponderação para cálculo da prevalência de anemia em nível municipal a fim de garantir uma amostra equiprobabilística. Para as crianças de 0-35, 9 meses, utilizando o critério de Brault-Dubuc, foi encontrada prevalência de anemia de $60,4 \%$, e para as crianças de 6-35, 9 meses, utilizando o critério da OMS (Hb $<11 \mathrm{~g} / \mathrm{dL}$ ), a prevalência foi de $54 \%$. Em relação ao sexo, foram encontradas nos meninos prevalências de $49,8 \%$ e $57,7 \%$ e nas meninas $58 \%$ e $63 \%$, respectivamente pelos critérios da OMS e de BraultDubuc. No modelo final de regressão logística, mantiveram significância estatística a idade da criança, com o maior risco na faixa etária de 12-18 meses, a renda familiar, inversamente associada à prevalência de anemia, e o número de pessoas por cômodo usado para dormir, aumentando-se o risco com o maior número de pessoas.

Em Salvador, Estado da Bahia, Assis et al.19 realizaram estudo transversal com o objetivo de identificar a prevalência e os fatores associados à anemia em uma amostra de 591 pré-escolares. A amostra foi estimada com base em uma prevalência de $20 \%$ para anemia e a seleção realizada seguindo uma amostragem em três estágios, correspondendo aos setores censitários, domicílios e crianças. A prevalência de anemia $(\mathrm{Hb}<11 \mathrm{~g} / \mathrm{dL})$ foi de $46,3 \%$. A análise de regressão logística mostrou maior risco de anemia para: idade de 6-12 meses; densidade de ferro na dieta $\leq 0,4 \mathrm{mg} / 100 \mathrm{Kcal}$; consumo de proteína $\leq 28,8 \mathrm{~g} / \mathrm{dia}$; indicador altura para idade $<-1$ escore$\mathrm{Z}$; e precárias condições físicas, ambientais e sanitárias do domicílio.

Analisando dados da II Pesquisa Estadual de Saúde e Nutrição do Estado de Pernambuco (II PESN-PE), Osório et al. 16 realizaram estudo transversal para investigar as possíveis razões para a alta prevalência de anemia em 650 crianças de 6-59 meses no Estado, selecionadas a partir de uma amostra representativa para a região metropolitana do Recife capital do Estado, interior urbano e interior rural. A amostragem sistemática foi realizada em três estágios: seleção dos municípios, dos setores censitários e dos domicílios, onde foram identificadas as crianças. Sete variáveis permaneceram significantes no modelo de regressão linear múltipla: idade, biodisponibilidade do ferro, retinol sérico, diarréia, tratamento da água de beber, condições sanitárias e baixo peso ao nascer, explicando $23,4 \%$ da variação da concentração de hemoglobina. 
Oliveira et al.,17 também trabalhando com o banco de dados da II PESN-PE, realizaram um estudo com o objetivo de identificar os fatores de risco da anemia em crianças de 6-59 meses, contemplando aspectos socioeconômicos e da dieta. Para tanto, foram utilizadas as informações do consumo alimentar de 746 crianças. A prevalência de anemia $(\mathrm{Hb}<11 \mathrm{~g} / \mathrm{dL})$ foi de $40,6 \%$. Na regressão logística permaneceram significantes as variáveis: proporção de calorias do leite de vaca, densidade de ferro nãoheme, idade e escolaridade materna. Ao analisar modelos por faixa etária, para as crianças menores de 24 meses, as variáveis significantes foram: a escolaridade materna e a densidade de ferro heme. Para as crianças de 24 meses ou mais permaneceram a proporção de calorias do leite de vaca e a densidade de ferro não-heme, como variáveis explicativas para a ocorrência de anemia.

Assunção et al.20 realizaram um estudo transversal de base populacional com uma amostra probabilística de 453 crianças com idade entre 0-5 anos, na zona urbana da cidade de Pelotas no Rio Grande do Sul, selecionadas a partir de amostragem por conglomerados em dois estágios: setores censitários e domicílios. A prevalência de anemia $(\mathrm{Hb}<11 \mathrm{~g} / \mathrm{dL})$ foi de $30,2 \%$, sendo de $27 \%$ no sexo masculino e $34 \%$ no feminino. Pela análise de regressão de Poisson, apenas a idade da criança e a renda familiar permaneceram no modelo como variáveis associadas à presença de anemia.

\section{Estudos estrangeiros}

Na Nova Zelândia, Soh et al.21 realizaram um estudo transversal em 243 crianças de 6-24 meses representativas de três cidades, Christchurch, Dunedin e Invercargill, a partir de uma amostragem randomizada em múltiplo estágio, incluindo as unidades censitárias e os domicílios. Os autores determinaram a prevalência da deficiência de ferro e os fatores associados com os níveis de ferritina. A prevalência de anemia $(\mathrm{Hb}<11 \mathrm{~g} / \mathrm{dL})$ foi de $49 \%$ e $22 \%$, respectivamente para as crianças de 6-11,9 e 12-24 meses. A prevalência de deficiência de ferro, diagnosticada pela presença de dois ou três parâmetros abaixo da normalidade $(\mathrm{VCM} \leq 73 \mathrm{fl}, \quad \mathrm{ZPP} \geq 70 \mu \mathrm{mol} / \mathrm{mol}$ e ferritina) foi de $4,3 \%$ e $5,6 \%$, considerando a ferritina $\leq 10 \mu \mathrm{g} / \mathrm{L}$ e $\leq 12 \mu \mathrm{g} / \mathrm{L}$, respectivamente. A anemia por deficiência de ferro esteve presente em $3,5 \%$ e $4,3 \%$ e a prevalência de depleção dos estoques de ferro foi de $12,6 \%$ e $18,6 \%$, respectivamente, considerando para ambos os critérios os níveis de ferritina $\leq 10 \mu \mathrm{g} / \mathrm{L}$ e $\leq 12 \mu \mathrm{g} / \mathrm{L}$. Em relação ao sexo, a prevalência da depleção dos estoques de ferro foi de $22,6 \%$ no sexo masculino e $13,3 \%$ sexo feminino. Em uma regressão linear múltipla, sexo feminino, etnia caucasiana e peso ao nascer foram positivamente associados com os valores da ferritina, enquanto que a idade e índice peso por idade, ajustados pelo peso ao nascer, foram negativamente associados à ferritina. Em um segundo modelo, contendo adicionalmente variáveis dietéticas, o consumo atual de fórmulas fortificadas com ferro e o consumo $>500 \mathrm{~mL}$ de leite de vaca por dia tiveram associação positiva e negativa, respectivamente, com a variação da ferritina.

Brotanek et al.22 realizaram um estudo com o objetivo de examinar a associação entre o uso prolongado de mamadeira e a deficiência de ferro em 2121 crianças de 1-3 anos, originada da amostragem populacional do National Health and Nutrition Survey III (NHANES III - USA). A prevalência de deficiência de ferro foi diagnosticada pela presença de dois ou três indicadores de acordo com a idade da criança: saturação de transferrina $<10 \%$, ferritina $<10 \mu \mathrm{g} / \mathrm{L}$ e protoporfirina eritrocitária livre $>1.42 \mu \mathrm{mol} / \mathrm{L}$, para crianças de $1-2$ anos; e saturação de transferrina $<12 \%$, ferritina $<10 \mu \mathrm{g} / \mathrm{L}$ e protoporfirina eritrocitária livre $>1.24 \mu \mathrm{mol} / \mathrm{L}$, para crianças de três anos. A deficiência de ferro foi encontrada em $9 \%$ das crianças. No sexo masculino a prevalência de deficiência de ferro foi de $9,2 \%$ e no sexo feminino foi de $8,8 \%$. Na análise de regressão logística multivariada apresentaram maiores risco de deficiência de ferro: crianças alimentadas por mamadeira entre 24-48 meses de idade quando comparadas àquelas $\leq 12$ meses, e crianças de etnia mexicana quando comparadas às de raça branca.

Um estudo desenvolvido no Vietnam por Nguyen et al. 23 determinou a distribuição e avaliou os fatores de risco para anemia em 9550 crianças menores de cinco anos. Para a seleção da amostra, o país foi dividido em sete zonas definidas de acordo com a semelhança geográfica e ecológica entre as províncias. A amostragem foi realizada em dois estágios: comunidades e domicílios em que existiam crianças menores de cinco anos, incluindo a criança mais jovem no estudo. A prevalência de anemia $(\mathrm{Hb}<11 \mathrm{~g} / \mathrm{dL})$ foi de $45,1 \%$. Na regressão logística multivariada sete fatores permaneceram significantes como preditores da anemia: ocupação materna (mães agricultoras); educação formal materna (sem educação formal); consumo de carne $(<1$ vez por semana); idade $(<24$ meses); morbidade (febre ou diarréia nas últimas duas semanas) e zonas ecológicas de moradia. Aproximadamente $17 \%$ da anemia foi atribuída à ocupação materna, como 
agricultora; $11 \%$ ao baixo consumo de carne; $8 \%$ à baixa educação materna; $3 \%$ a febre e $2 \%$ a diarréia nas 2 últimas semanas.

Brotanek et al., ${ }^{24}$ utilizando uma amostra originada do National Health and Nutrition Survey IV (NHANES IV - USA) identificaram os fatores de risco para deficiência de ferro utilizando dados de 960 crianças de 1-3 anos que possuíam resultados laboratoriais para os três indicadores de estado do ferro (saturação de transferrina, ferritina e protoporfirina eritrocitária livre). A deficiência de ferro, considerando os pontos de corte do NHANES III USA acima referidos, 22 foi de $8 \%$, sendo $8,2 \%$ no sexo masculino e de $7,6 \%$ no sexo feminino. No modelo multivariado final, a deficiência de ferro apresentou-se significantemente associada ao acesso à creche e ao indicador de peso para altura, sendo os maiores riscos, respectivamente, para crianças sem acesso à creche e crianças com sobrepeso.

Ngnie-Teta et al.,25 analisando os dados da pesquisa de demografia e saúde, com amostras representativas nacionais de 564 e 774 crianças de 659 meses de Benin e Mali, respectivamente, identificaram os fatores de risco para anemia em nível individual, domiciliar e comunitário através de um estudo transversal utilizando regressão multivariada e multinível. A amostragem foi do tipo probabilística realizada em dois estágios: seleção das áreas seguida pelos domicílios e indivíduos. As prevalências de anemia $(\mathrm{Hb}<11 \mathrm{~g} / \mathrm{dL})$ foram de $82 \%$ e $83 \%$ em Benin e Mali, respectivamente. As crianças do sexo masculino apresentaram prevalências de anemia de $84,1 \%$ e $82,3 \%$ e as do sexo feminino de $80,3 \%$ e $84,2 \%$, respectivamente em Benin e Mali. Nas análises multinível e multivariada foi utilizada a anemia moderada e severa $(\mathrm{Hb}<9,9 \mathrm{~g} / \mathrm{dL})$ como variável resposta, em ambos os países. Em Benin a anemia mostrou associação com os seguintes fatores: idade (o risco de anemia foi maior nas crianças menores de três anos do que naquelas de 4-5 anos), ordem de nascimento (crianças com ordem de nascimento maior que cinco tiveram risco maior), o indicador altura para idade (maior risco em crianças com baixa estatura), diarréia (maior risco em crianças com história recente de diarréia), imunização (maior risco em crianças com imunização incompleta), o uso de mosquiteiro (maior risco naquelas que não faziam uso da peça), baixo nível de educação materna e baixo índice de desenvolvimento da comunidade. Em Mali, os fatores determinantes da anemia foram: idade (maior risco nas crianças de 12 a 35 meses), indicador altura para idade (maior risco em crianças com baixa estatura), diarréia (maior risco em crianças com história recente de diarréia), índice de enriquecimento do domicílio (maior risco para domicílios com médio enriquecimento) e local de residência (maior risco em área rural). A contribuição dos fatores relacionados à comunidade foi de $14 \%$ e $19 \%$, respectivamente em Benin e Mali. Na Jordânia, Hong et al.,26 através de um estudo transversal de representação nacional (Pesquisa Populacional de Saúde Familiar da Jordânia), examinaram os efeitos do fumo passivo na anemia em uma subamostra de 740 crianças de 0-35 meses de idade, referente a $27 \%$ da amostra total, que tinha resultado da hemoglobina. A amostragem foi realizada em múltiplos estágios, estratificada por províncias e áreas urbana e rural. A prevalência de anemia $(\mathrm{Hb}<10,0 \mathrm{~g} / \mathrm{dL})$ foi de $19 \%$, sendo de $18,7 \%$ no sexo masculino e de $20 \%$ no sexo feminino. A regressão logística multivariada evidenciou que crianças expostas ao fumo passivo por ambos os pais tinham três vezes mais chances de ter anemia do que aquelas não expostas. Esta associação permaneceu significante mesmo quando a idade, o sexo, a ordem de nascimento, o peso ao nascer, o aleitamento materno e o status nutricional foram controlados e também quando foram controladas as variáveis maternas (suplementação de ferro durante a gravidez, idade ao nascimento da criança, presença de anemia, índice de massa corporal e educação). No modelo final, quando controlados todos os fatores para crianças, para a mãe, para o domicílio (status de riqueza do domicílio, qualidade da água de beber, toaletes com boas condições higiênicas) e regionais (regiões geográficas), as crianças jovens expostas ao fumo passivo de ambos os pais permaneceram significantemente mais prováveis de serem anêmicas. A desnutrição severa foi um fator de alto risco para a anemia, independentemente do tabagismo, fumo passivo e de outros fatores de risco. Por outro lado, outros fatores se mostraram associados ao menor risco de anemia em crianças: de maior idade, de mães acima de 34 anos e domicílios com toaletes em boas condições higiênicas.

Com o objetivo de avaliar o status nutricional e a prevalência de anemia nas crianças da Guiné Equatorial, Custodio et al. ${ }^{27}$ conduziram um estudo transversal com amostra representativa nacional de 524 crianças menores de cinco anos. O processo de amostragem foi realizado randomicamente em múltiplos estágios, considerando-se as unidades amostrais primárias (aldeias na área rural e localidades urbanas vizinhas), os domicílios e as crianças, garantindo a proporcionalidade de acordo com o Censo Populacional de domicílios de 1994. A prevalência de anemia $(\mathrm{Hb}<11 \mathrm{~g} / \mathrm{dL})$ foi de $69,3 \%$ e de anemia moderada ou severa $(\mathrm{Hb}<8 \mathrm{~g} / \mathrm{dL})$ foi de $8,3 \%$, sendo 
esta última de $84,3 \%$ no sexo masculino e de $45,7 \%$ no sexo feminino. Uma regressão logística multivariada para a anemia moderada ou severa evidenciou que o alto nível socioeducacional no domicílio foi negativamente associado e a paridade materna acima de cinco filhos positivamente associada à anemia em crianças.

Brotanek et al.28 analisaram a tendência secular da deficiência de ferro em crianças americanas de um a três anos de idade, utilizando dados de amostras populacionais de 2201, 3875 e 1641 crianças dos National Health and Nutrition Surveys II, III e IV (NHANES II, III e IV - USA), respectivamente. O diagnóstico da deficiência de ferro foi confirmado quando dois ou três indicadores do status do ferro apresentaram os seguintes níveis: saturação de transferrina $<10 \%$, ferritina $<10 \mu \mathrm{g} / \mathrm{L}$ e protoporfirina eritrocitária livre $>1.42 \mu \mathrm{mol} / \mathrm{L}$, para crianças de 1-2 anos; e saturação de transferrina $<12 \%$, ferritina $<10 \mu \mathrm{g} / \mathrm{L}$ e protoporfirina eritrocitária livre $>1.24 \mu \mathrm{mol} / \mathrm{L}$, para crianças de 3 anos. Especificamente para os dados do NHANES II, a ferritina sérica foi substituída pelo volume corpuscular médio (MCV), tomando-se como critérios os níveis $<73 \mathrm{fL}$ para crianças de 3 anos e $<75 \mathrm{fL}$ para crianças de dois anos. Os resultados não evidenciaram mudanças estatisticamente significantes na prevalência da deficiência de ferro entre 1976 e 2002. A deficiência de ferro foi de $10 \%, 9,1 \%$ e $8 \%$ nas crianças de 1-3 anos nos II, III e IV inquéritos, respectivamente. Em relação ao sexo essas prevalências foram de $13,3 \%, 9,3 \%$ e $8,2 \%$ no masculino e $6,6 \%, 8,8 \%$ e $7,8 \%$ no feminino, nos II, III e IV inquéritos, respectivamente. $\mathrm{Na}$ análise de regressão logística multivariada apresentaram maior risco de deficiência de ferro: crianças de etnia hispânica; crianças que viviam em domicílios com renda familiar abaixo da linha de pobreza; e, crianças com sobrepeso. A idade de 2-3 anos foi negativamente associada à deficiência de ferro, quando comparadas as crianças de 1 ano. Após ajuste pelo idioma falado no domicílio (inglês ou não), a renda familiar não manteve sua significância estatística.

No Timor-Leste, Agho et al.29 avaliaram a prevalência de anemia e os fatores associados à concentração de hemoglobina em 4514 crianças de 6-59 meses de idade. Foram utilizados os dados de um inquérito de demografia e saúde realizado em 2003, por meio de uma amostragem multiestágios, estratificada por área geográfica (urbana, oeste rural, centro rural e leste rural), onde foram selecionadas as vilas, subvilas e os domicílios, respectivamente. A prevalência de anemia $(\mathrm{Hb}<11 \mathrm{~g} / \mathrm{dL})$ foi $38,2 \%$ em crianças de 6-23 meses e 22,2\% naquelas de 24-59 meses de idade. A análise de regressão linear múltipla evidenciou associação positiva da idade da criança e do aleitamento materno até o sexto mês de vida com a concentração de hemoglobina. As seguintes variáveis foram negativamente associadas à concentração de hemoglobina: peso $\mathrm{x}$ altura $<-2 \mathrm{SZ}$, sexo masculino, presença de diarréia nas últimas duas semanas, viver em domicílios de classe média, anemia materna e possuir mães com maior nível educacional. Com relação a esta última associação, os autores referem que, no Timor-Leste, as piores práticas de aleitamento materno ocorrem nos grupos de melhor condição socioeconômica.

Subramanian et al.30 investigaram a altura materna e sua associação com a anemia na India em uma amostra de 36.549 crianças de 6-59 meses, provenientes do National Family Health Survey (NFHS), realizado em 2005-2006. A amostragem em múltiplos estágios foi estratificada por área de residência (rural e urbana) e garantiu a representatividade da população. A anemia $(\mathrm{Hb}<11 \mathrm{~g} / \mathrm{dL})$ foi diagnosticada em $69,1 \%$ e anemia grave $(\mathrm{Hb}<7 \mathrm{~g} / \mathrm{dL})$ em $2,7 \%$ das crianças. Foi realizada a análise de regressão de Poisson com erro robusto. Os modelos finais para anemia e anemia grave, não evidenciaram a altura materna como fator determinante para a anemia.

\section{Discussão}

De fato, investigar os fatores determinantes da anemia e/ou deficiência de ferro nas crianças é considerado um tema de relevância em saúde pública e existem inúmeras publicações direcionadas a esse fim. Entretanto, observa-se que, proporcionalmente ao número de publicações encontradas, são poucas aquelas que abordam o problema a partir de amostras populacionais representativas.

Um aspecto importante a ser levado em consideração, ao verificar os estudos incluídos nessa revisão, é a falta de uma padronização para definição sobre quais parâmetros e respectivos pontos de corte seriam mais adequados para o diagnóstico da anemia e da deficiência de ferro. Em doze estudos, $14,15,17$ $21,23,25,27,29,30$ foi utilizado o ponto de corte indicado pela Organização Mundial da Saúde 1,2 para o diagnóstico da anemia $(\mathrm{Hb}<11 \mathrm{~g} / \mathrm{dL}$, para crianças de 659 meses), entretanto, um dos estudos internacionais 26 utilizou o ponto de corte da hemoglobina $<10 \mathrm{~g} / \mathrm{dL}$. Em relação à deficiência de ferro, nenhum estudo brasileiro avaliou esta deficiência e os estudos estrangeiros que o fizeram, utilizaram dois ou mais parâmetros associados, como ferritina, saturação da transferrina, protoporfirina eritrocitária 
livre, zincoprotoporfirina e volume corpuscular médio. $21,22,24,28$ A ferritina isoladamente foi utilizada em um estudo para diagnosticar a depleção dos estoques de ferro. ${ }^{21}$

Outro aspecto a ser observado, é a grande diversidade de variáveis analisadas. Elas são diferentes para cada estudo, dependendo da hipótese a ser investigada, do contexto ambiental, socioeconômico e cultural da população e da viabilidade da pesquisa. Assim, não é simples explicar os fatores determinantes da anemia e fazer uma análise generalizada sobre a contribuição específica de cada determinante no aparecimento da anemia, limitando a validade externa desses estudos. ${ }^{14-21,24-26}$

Pode-se ainda considerar limitante o fato de que os artigos aqui analisados são estudos transversais, os quais além de estarem sujeitos a vários vieses, permitem observar apenas associações entre os eventos, impossibilitando determinar a relação de causa e efeito entre estes. Outra limitação da presente revisão foi a utilização apenas dos artigos em inglês ou português, o que impossibilitou o acesso a alguns resultados originados de revistas publicadas em outros idiomas.

Entre os estudos aqui apresentados, foram encontrados quarenta e dois fatores causais. Os mais citados como fatores estatisticamente associados à anemia ou a deficiência de ferro foram: idade da criança, $16-21,23,25,26,28,29$ escolaridade materna, 14,17,23,25 renda familiar, 14,18,20 diarréia, 16,23,25 área geográfica,23,25 índice de riqueza do domicílio,25,29 peso ao nascer, 16,21 indicador altura/idade, 19,25,27,29 indicador peso/altura, 24,29 etnia, 21,22 sexo, 21,29 densidade de ferro 14,19 e calorias provenientes do leite de vaca. 15,17

A partir do levantamento acima se percebe um grande número de fatores determinantes encontrados. Entretanto, ao analisar a presença da mesma variável nos diferentes estudos, verifica-se que apenas algumas delas se apresentaram como determinantes expressivos para a ocorrência da anemia. Dos trabalhos que analisaram a idade da criança, ${ }^{16}$ 29 onze estudos 16-21,23,25,26,28,29 demonstraram associação desta variável com o risco de ocorrência da anemia ou diminuição da concentração de hemoglobina. Com relação à área geográfica, 16,23,25,26 esta se apresentou como fator de risco em dois estudos. 23,25 A etnia $21,22,24$ também esteve associada ao risco de anemia em 2 estudos.21,22 Houve consenso entre os estudos que avaliaram a presença de diarreia 16,23,25,29 e os fatores dietéticos, como a densidade de ferro 14,19 e o percentual de calorias provenientes do leite de vaca, 15,17 de que estas variáveis estariam associadas ao maior risco de anemia. As demais variáveis, que se mostraram estatisticamente associadas à anemia ou a deficiência de ferro, não se apresentaram como fator de risco na maioria dos estudos onde foram avaliadas, dificultando a compreensão da influência destes fatores causais na determinação do problema.

Apesar do conhecimento de que os fatores associados à anemia variam de acordo com características geográficas, nível de desenvolvimento e condições socioeconômicas, ${ }^{2}$ a idade da criança, atrelada muito mais às condições fisiológicas do crescimento infantil do que aos determinantes socioeconômicos e ambientais, foi apresentada como a variável mais comumente associada à anemia, independentemente do grau de desenvolvimento da região pesquisada.

Ao considerar as diferenças socioeconômicas entre as regiões de desenvolvimento dos estudos, chama a atenção o fato de que a anemia esteve associada ao déficit nutricional nos países em desenvolvimento 19,25,26 e, contrariamente, ao sobrepeso nos países desenvolvidos. 21,24

No Brasil, a transição nutricional que vem se desenhando no momento atual aponta para a redução do déficit estatural e aumento na prevalência de sobrepeso.3,31 Contudo, nos estudos nacionais aqui reportados ainda não se encontram dados referentes à associação entre anemia e sobrepeso nas crianças menores de seis anos, lacuna que necessita ser preenchida tendo em vista as especificidades nas estratégias de controle da anemia para as diferentes situações.

Outros fatores que se mostraram associados à anemia em crianças menores de seis anos apenas em países em desenvolvimento foram a escolaridade materna $14,17,23,25$ e as condições de moradia e sanitárias. 16,19,25-26

Considerando a distribuição global da anemia, tanto em países desenvolvidos quanto nos países em desenvolvimento, a Organização Mundial da Saúde, em revisão sistemática de estudos de 1993-2005, determinando as prevalências de anemia em diferentes regiões, estima que $47,4 \%$ das crianças préescolares são anêmicas no mundo. ${ }^{2}$ Propor estratégias para modificar esse cenário, tomando por base as características inerentes a cada região, torna-se uma tarefa difícil, em virtude da existência de poucos estudos representativos sobre os fatores associados à anemia.

As diferenças observadas no comportamento das variáveis pesquisadas entre países desenvolvidos e em desenvolvimento, a diversidade dos fatores investigados em cada estudo e as pesquisas com novas variáveis, como a exposição ao passiva ao 
fumo e a obesidade, apontam para a necessidade de realização de estudos populacionais com uma quantidade de variáveis explanatórias comuns aos diferentes estudos. Isto possibilitaria uma compreensão mais abrangente do processo da instalação da anemia e/ou deficiência de ferro nas crianças menores de seis anos, considerando os diferentes contextos

\section{Referências}

1. World Health Organization. Iron deficiency anaemia Assessment, prevention and control. A guide for programme managers, Geneva; 2001; 114p.

2. World Health Organization. Worldwide prevalence of anaemia 1993-2005: WHO global database on anaemia, Geneva; 2008; 41p.

3. Batista Filho M, Rissin A. A transição nutricional no Brasil: tendências regionais e temporais. Cad. Saúde Pública. 2003; 19 ( Supl. 1): 181-91.

4. Saloojee H, Pettifor JM. Iron deficiency and impaired child development. Br Med J. 2001; 323: 1377-8.

5. Stoltzfus RJ. Iron-deficiency anemia: reexamining the nature and magnitude of the public health problem. Summary: implications for research and programs. J Nutr. 2001; 131(Suppl): 697S-701S.

6. Assunção MCF, Santos IS. Efeito da fortificação de alimentos com ferro sobre anemia em crianças: um estudo de revisão. Cad. Saúde Pública. 2007; 23: 269-81.

7. Bortolini AG, Vitolo MR. Baixa adesão à suplementação de ferro entre lactentes usuários de serviço público de saúde. Pediatr (São Paulo). 2007; 29: 176-82.

8. Mora JO. Forging effective strategies to combat iron deficiency. Iron supplementation: overcoming technical and practical barriers. J Nutr. 2002; 132 (Suppl): 853S-5S.

9. Yip R. Forging effective strategies to combat iron deficiency. Prevention and control of iron deficiency: policy and strategy issues. J Nutr. 2002; 132 (Suppl): 802S-5S.

10. Stoltzfus RJ. Iron-deficiency anemia: reexamining the nature and magnitude of the public health problem. Defining Iron-deficiency anemia in public health terms: a time for reflection. J Nutr. 2001; 131 (Suppl): 565S-7S

11. Curso de revisão sistemática e metanálise. 2007. Disponível em: http://www.virtual.epm.br/cursos/metanalise/conteudo/ valida.php.

12. Liberati A, Altman DG, Tetzlaff J, Mulrow C, Gotzsche PC, Loannidis JPA, Clarke M, Devereaux PJ, Kleijnen J, Moher D. The PRISMA statement for reporting systematic reviews and meta-analyses of studies that evaluate health care interventions: explanation and elaboration. Ann Intern Med. 2009; 151: 65-93.

13. DECS (Descritores em Ciências da Saúde). 2007. Disponível em: http:/www.decs/bvs/br

14. Monteiro CA, Szarfarc SC, Mondini L. Tendência secular da anemia na infância na cidade de São Paulo (1984-1996). Rev Saúde Pública. 2000; 34 (Supl. 6): 62-72.

15. Levy-costa RB, Monteiro CA. Consumo de leite de vaca e anemia na infância no Município de São Paulo. Rev Saúde ambiental, cultural, socioeconômico, de saúde e nutrição de cada população. A multicausalidade do problema requer estratégias com ações integradas de prevenção e tratamento, de acordo com as características específicas de cada população, considerando seus principais fatores etiológicos.

Pública. 2004; 38: 797-803.

16. Osório MM, Lira PIC, Ashworth A. Factors associated with $\mathrm{Hb}$ concentration in children aged 6-59 months in the State of Pernambuco, Brazil. Br J Nutr. 2004; 91: 307-14.

17. Oliveira MAA, Osório MM, Raposo MCF. Socioeconomic and dietary risk factors for anemia in children aged 6 to 59 months. J Pediatr. 2007; 83: 39-46.

18. Neuman NA, Tanaka OU, Szarfarc SC, Guimarães PRV, Victora CG. Prevalência e fatores de risco para anemia no Sul do Brasil. Rev Saúde Pública. 2000; 34: 56-63.

19. Assis AMO, Barreto ML, Gomes GSS, Prado MS, Santos NJ, Santos LMP, Sampaio LR, Ribeiro RC, Oliveira LPM, Oliveira VA. Childhood anemia prevalence and associated factors in Salvador, Bahia, Brazil. Cad Saúde Pública. 2004; 20: 1633-41.

20. Assunção MCF, Santos IS, Barros AJD, Gigante DP, Victora CG. Anemia em menores de seis anos: estudo de base populacional em Pelotas, RS. Rev Saúde Pública. 2007; 41: 328-35

21. Soh P, Ferguson EL, McKenzie JE, Homs MYV, Gibson RS. Iron deficiency and risk factors for lower iron stores in 6-24 month-old New Zealanders. Eur J Clin Nutr. 2004; 58: 71-9.

22. Brotanek JM, Halterman JS, Auinger P, Flores G, Weitzman M. Iron deficiency, prolonged bottle-feeding, and racial/ethnic disparities in young children. Arch Pediatr Adolesc Med. 2005; 159: 1038-42.

23. Nguyen PH, Nguyen KC, Le Mai B, Nguyen TV, Ha KH, Bern C, Flores R, Martorell R. Risk factors for anemia in Vietnam. Southeast Asian J Trop Med Public Health. 2006; 37: 1213-23.

24. Brotanek JM, Gosz J, Weitzman M, Flores G. Iron deficiency in early childhood in the United States: risk factors and racial/ethnic disparities. Pediatrics. 2007; 120: 568-75.

25. Ngnie-Teta I, Receveur O, Kuate-Defo B. Risk factors for moderate to severe anemia among children in Benin and Mali: Insights from a multilevel analysis. Food Nutr Bull. 2007; 28: 76-88.

26. Hong R, Betancourt JA, Ruiz-Beltran M. Passive smoking as a risk factor of anemia in young children aged 0-35 months in Jordan. BMC Pediatrics. 2007; 7: 16.

27. Custodio E, Descalzo MA, Roche J, Sánchez I, Molina L, Lwanga M, Bernis C, Villamor E, Baylin A. Nutritional status and its correlates in Equatorial Guinean preschool children: Results from a nationally representative survey. Food Nutr Bull. 2008; 29: 49-58. 
28. Brotanek JM, Gosz J, Weitzman M, Flores G. Secular trends in the prevalence of iron deficiency among US toddlers, 1976-2002. Arch Pediatr Adolesc Med. 2008; 162: 374-81.

29. Agho KE, Dibley MJ, D’Este C, Gibberd R. Factors associated with haemoglobin concentration among Timor-Leste children aged 6-59 months. J Health Popul Nutr. 2008; 26: $200-9$

Recebido em 29 de abril de 2010

Versão final apresentada em 23 de novembro de 2010

Aprovado em 30 de novembro de 2010
30. Subramanian SV, Ackerson LK, Smith GD, John NA. Association of maternal height with child mortality, anthropometric failure and anemia in India. JAMA. 2009; 301: 1691-701.

31. Batista Filho M, Souza AI, Miglioli TC, Santos MC. Anemia e obesidade: um paradoxo da transição nutricional brasileira. Cad Saúde Pública. 2008; 24 (Supl. 2): S247-57. 